\title{
CHARACTERISTIC FUNCTIONS OF LIFTINGS-II
}

\author{
Santanu Dey, Rolf Gohm And Kalpesh J. HaRia
}

Abstract. We prove that the symbol of the characteristic function of a minimal contractive lifting is an injective map and that the converse also holds, using explicit computation and functional models. We discuss when the characteristic function of a lifting is a polynomial and present a series representation for the characteristic functions of liftings.

Mathematics subject classification (2010): 47A20, 47A13, 47A15, 47A48, 81R15.

Keywords and phrases: Row contraction, minimal contractive lifting, characteristic function, multianalytic operator, completely non-coisometric, linear system, transfer function.

\section{REFERENCES}

[1] J. A. BALl, G. GROENEWALD AND T. MALAKorn, Conservative structured noncommutative multidimensional linear systems. The state space method generalizations and applications, 179-223, Oper. Theory Adv. Appl. 161, Birkhäuser, Basel (2006).

[2] J. BALL AND V. VinNikov, Lax-Phillips scattering and conservative linear systems: a Cuntz-algebra multidimensional setting, Mem. Amer. Math. Soc., 178 (2005).

[3] S. Dey And R. Gohm, Characteristic functions of liftings, J. Operator Theory, 65 (2011), 17-45.

[4] S. DeY, R. Gohm AND K. J. HARIA, Functional models and minimal contractive liftings, Complex Analysis and Operator Theory, 9 (2015), 933-955.

[5] S. Dey, K. J. HaRIA, Generalized repeated interaction model and transfer functions. Operator theory in harmonic and non-commutative analysis, 111-135, Oper. Theory Adv. Appl., 240, Birkhäuser/Springer, Cham, 2014.

[6] C. FoiAs AND J. SARKAR, Contractions with polynomial characteristic functions I. Geometric approach, Trans. Amer. Math. Soc. 364 (2012), 4127-4153.

[7] R. GoHM, Non-commutative Markov chains and multi-analytic operators, J. Math. Anal. Appl., 364 (2010), 275-288.

[8] R. GoHM, Transfer functions for pairs of wandering subspaces, Spectral Theory, Mathematical System Theory, Evolution Equations, Differential and Difference Equations, Series: Operator Theory: Advances and Applications, 221, Birkhäuser (2012), 385-398.

[9] K. J. HARIA, Outgoing Cuntz scattering system for a coisometric lifting and transfer function, Proc. Indian Acad. Sci. Math. Sci. 123 (2013), 427-442.

[10] G. POPESCU, Isometric dilations for infinite sequences of noncommuting operators, Trans. Amer. Math. Soc. 316 (1989), 523-536.

[11] G. PopesCu, Characteristic functions for infinite sequences of noncommuting operators, J. Operator Theory 22 (1989), 51-71.

[12] G. PopesCU, Unitary invariants on the unit ball of $B(\mathscr{H})^{n}$, Trans. Amer. Math. Soc. 365 (2013), 6243-6267. 